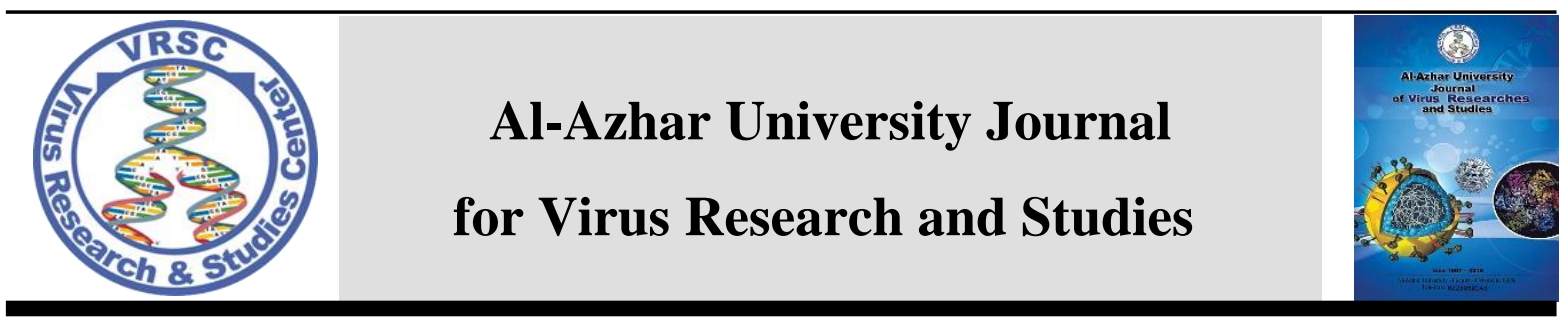

\title{
Study on Serum Cholinesterase as Marker of Chronic
}

\author{
Liver Disease
}

\author{
Laila M Aboughazala, Fatma M Anan* \\ Center for Virus Research and Studies, Al-Azhar University, Cairo, Egypt \\ *E-mail: vrsc@azhar.edu.eg
}

\begin{abstract}
Chronic liver disease is marked by the gradual destruction of liver tissue over time and replacement of normal liver with nodules of scar tissue. Several liver diseases fall under this category. The end results of the gradual destruction are cirrhosis and fibrosis of the liver. It is estimated that more than 170 million people worldwide are chronically infected with the hepatitis $\mathrm{C}$ virus $(\mathrm{HCV})$. The most important consequence of chronic $\mathrm{HCV}$ infection is progressive liver fibrosis leading to cirrhosis and finally to hepatocellular carcinoma (HCC), which has significant morbidity and mortality. Many factors, such as alcohol intake, older age at time of infection, male gender, and co-infection with the human immunodeficiency virus or hepatitis B virus accelerate disease progression. There are two types of cholinesterase enzymes in the blood: acetylcholinesterase, found mainly in red blood cells (RBC) and butarylcholinesterase found in serum or plasma. The general term $\mathrm{ChE}$ is used for both enzymes MicroRNA (miRNA) was first discovered in 1993, and was characterized as a small, non-coding RNA miRNA are small RNA molecules which target many mRNA transcripts, leading to their post-transcriptional silencing. Many mRNAs can be silenced by multiple miRNA and miRNA often target more than one mRNA participating in a particular biological function (MicroRNA-132 (miR-132) targets acetylcholinesterase (AChE) and potentiates the cholinergic blockade of inflammatory reactions in cultured cells
\end{abstract}

Keywords: Chronic liver disease, hepatitis $\mathrm{C}$ virus, red blood cells.

\section{Introduction}

The liver is a very important organ present in vertebrates and other animals. It has a many of functions, such as detoxification, protein synthesis, and production of biochemicals necessary for digestion. The liver is important for survival, there is currently no way to compensate for the absence of liver function. The liver plays a major role in metabolism and have many functions in the body, including glycogen storage, decomposition of red blood cells, plasma protein synthesis, hormone production, and detoxification. It lies below the diaphragm in the thoracic region of the abdomen. Liver produces bile, an alkaline compound which aids in digestion, via the emulsification of lipids. The liver's highly specialized tissues regulate a wide variety of high-volume biochemical reactions, including the synthesis and breakdown of 
small and complex molecules, many of which are necessary for normal vital functions (9).

\section{Liver diseases:}

Liver diseases may be occurred by various kinds of causes as: drug, alcohol intake and viruses. Viral infections are the most common diseases that affect the liver, when the liver cell damaged by viruses, the cell can become with no function, with fewer healthy cells to carry on their important work, most of body functions can be affected. According to the office for national statistics in the United Kingdom, liver disease is now the fifth most common cause of death after heart disease, stroke, chest disease and cancer. however, unlike other major causes of mortality, liver disease rates are increasing rather than declining. a recent UK study showed that mortality rates in Scotland for subjects with cirrhosis have more than doubled for men and have increased by almost half for women (10).

\section{Chronic liver disease:}

Chronic disease constitutes a fastincreasing burden to society. The World Health Organization (WHO) evaluates that $46 \%$ of global disease and $59 \%$ of mortality is because the chronic diseases. Thirty-five million persons in the world die each year due to infection by chronic disease and the numbers are increasing every year. The main functions of liver failed when a liver develops cirrhosis, cirrhosis is called pathologically by the loss of normal microscopic lobular architecture with fibrosis and nodular regeneration. One of the most cause of death in the world is chronic liver diseases and cirrhosis. Chronic liver disease, particularly in the end stage, is characterized by clinical bleeding and decreased levels of most procoagulant factors (11).

\section{Causes:}

The list of conditions associated with chronic liver disease is extensive and can be categorized in the following way:

Viral causes:

- Hepatitis B

- Hepatitis C

Toxic and drugs:

- Alcoholic liver disease

Metabolic:

- Non-alcoholic fatty liver disease

Liver fibrosis occurred after chronic damage of the liver. The main causes of liver fibrosis developed because infection by chronic hepatitis $\mathrm{B}$ and $\mathrm{C}$ virus (HBC, $\mathrm{HCV}$ ) infection, alcoholic and nonalcoholic steatohepatitis. It is becoming increasingly clear that hepatocyte apoptosis contributes to liver fibrogenesis. Recent studies of chronic HBV and HCV infection as well as non-alcoholic fatty liver disease (NAFLD) show that hepatocyte apoptosis correlates with disease severity and stage of fibrosis. Acute liver failure (ALF) occurs when rapid death or injury to a large proportion of hepatocytes, leaving insufficient hepatic parenchymal mass to sustain liver function (12).

\section{Viral causes:}

In the United States, about 150,000 people in the next 10 years will die from liver cancer and end-stage liver disease associated with chronic hepatitis $\mathrm{B}$ and hepatitis $\mathrm{C}$, because of the asymptomatic 
nature of chronic hepatitis $\mathrm{B}$ and hepatitis $\mathrm{C}$, the people infected with $\mathrm{HBV}$ and $\mathrm{HCV}$ are not aware that they have been infected until they have symptoms of cirrhosis or a type of liver cancer, hepatocellular carcinoma (HCC), HCV is efficiently transmitted by direct percutaneous exposure to infectious blood. Persons who received a blood transfusion before 1992 and past or current injection-drug users, they have chronic HCV infection include those. After a person is exposed to HBV or HCV acute form occurs within the first 6 months. Chronic hepatitis $\mathrm{B}$ and chronic hepatitis $\mathrm{C}$ can result in liver cirrhosis and a type of liver cancer (13).

\section{Hepatitis B:}

Hepatitis B virus (HBV) is a noncytopathic, hepatotropic virus of the Hepadnaviridae family that causes variable degrees of liver disease in humans. When person infected with HBV, the infection may be acute or chronic, while adult infections have a relatively low rate of chronicity (around $5 \%$ ), neonatal infections usually have a high persistence rate.( $(\varepsilon)$ Chronic infection is mostly asymptomatic, but HBV carriers are at risk of developing lifethreatening cirrhosis and later on hepatic carcinoma (15). Hepatitis B surface antigen (HBsAg) is considered a milestone in the research history of hepatitis $\mathrm{B}$ virus (HBV) and has been used for more than 40 years to confirm HBV infections.( ( 7 )

The second generation of standard HBsAg samples introduced by the WHO, quantitative $\mathrm{HBsAg}$ tests have been performed $(17 ; 18)$.

\section{HBV transmission:}

HBV can be transmitted by sexual contact, through the skin, by inoculation with contaminated blood or blood products, by transplantation of organs from infected donors, and perinatally from infected mothers. Serum hepatitis B surface antigen (HBsAg) and HBV DNA are considered reliable indicators of active infection (19).

\section{HBV life cycle:}

Hepatitis B virus (HBV) has unique life cycle that results in the production of enormous viral loads during active replication without actually killing the infected cell directly Because HBV uses reverse transcription to copy its DNA genome, mutant viral genomes emerge frequently (20).

During both acute and persistent infection, high levels of infectious HBV (virions) circulate in the bloodstream. Hepatocytes, the major targets of the virus, are separated from the bloodstream by endothelial and Kupffer cells that line the sinusoids of the liver $(21 ; 22)$.

\section{Symptoms and signs of chronic viral infection hepatitis:}

Chronic viral hepatitis occure with asymptomatic nature of liver disease at most people and the slowness or absence of progression to advanced liver disease. The HBV infection with no symptoms and no clinical signs, symptoms and signs of chronic viral hepatitis can be divided into those associated with:

- Early or slowly progressive liver disease

- Progressive liver disease

- Advanced liver disease complications

- Extrahepatic manifestations

In this classification, 'early or slowly progressive liver disease' includes people with chronic hepatitis $\mathrm{C}$ who progress 
slowly and may have early fibrosis. 'Progressive liver disease' covers people who progress to cirrhosis or, in the case of chronic HBV infection, have clinical evidence of progressive disease such as hepatitis 'flares' but retain adequate liver function (e.g. compensated cirrhosis). 'Advanced liver disease intricacy' includes people with clinical liver failure (HCC) (23).

\section{Vaccines:}

Worlds have a developed vaccine to prevent hepatitis B infection, but although Hepatitis B control has not been attained yet (23). In fact, many people carriers and the burden of their disease remains, so that efforts must be continuing to treat the various stages of disease. $\mathrm{HB}$ vaccine is the first and the only vaccine against a major human cancer. Vaccination is the most effective tool in preventing the transmission of HBV. hepatitis B vaccine induces protection in about $95 \%$ of recipients. A safe and effective vaccine against HBV infection has been available for 20 years. $H B$ vaccine is effective in preventing HBV infections when it is given either before exposure or shortly after exposure, At least $85 \%-90 \%$ of HBVassociated deaths are vaccine-preventable. Despite the availability of a vaccine, worldwide infection persists (24).

\section{Hepatitis C:}

In 1980, hepatitis C virus (HCV) was discovered. After 25 years, new highly active antiviral drug combinations are reaching the Western world's markets, helping for possibly therapeutic, the discovery of $\mathrm{HCV}$, one of the most important of the 20th century, that used an original approach based on molecular biology methods, subsequently used to discover a large number of other viruses
(25). Still hepatitis $\mathrm{C}$ virus (HCV) infection is a major health burden affecting 130 to 170 million people worldwide. Most infections keep and continuously infected people have a high risk to develop liver cirrhosis and hepatocellular carcinoma after 10 to 30 years of infection (26). Most people infected with the virus are unaware of their infection and, for many who have been diagnosed, treatment remains unavailable (27).

\section{HCV transmission:}

\section{Healthcare associated transmission}

Before 1992, many people infected with $\mathrm{HCV}$ through blood product transfusions. In 1992, a reliable blood test to identify HCV antibodies became available. Then, the blood supply has been screened. Now the risk is considered to be less than 1 chance per 2 million units of transfused blood (28). Hepatitis C virus infection is strongly associated with health inequity; in low- and middle-income countries, infection with $\mathrm{HCV}$ is most commonly associated with unsafe injection practices and procedures such as renal dialysis and unscreened blood transfusions $(29 ; 30)$. Between 8 and 12 billion injections are administered yearly around the world and $50 \%$ of these are considered to be unsafe (mainly in sub-Saharan Africa and Asia) (31).

\section{People who inject drugs}

The most infections of HCV in middle- and high-income countries, happen when people use unsterile equipment to inject drugs and contaminated drug solutions. Of the estimated 16 million people in 148 countries who actively inject drugs, 10 million are infected with HCV (32). 


\section{Mother-to-child transmission}

Low risk of transmission of $\mathrm{HCV}$ to babies from their mother, this occurs in $4-8 \%$ of births to women with only HCV infection and the risk increase to $17-25 \%$ of births to women infected with $\mathrm{HIV}$ and $\mathrm{HCV}$ coinfection (33).

\section{Sexual transmission}

Sexual transmission of HCV occurs infrequently in heterosexual couples (34).

\section{Other}

Many other routes of HCV transmission such as intranasal drug use and other modes of bloodborne transmission, as acquisition by health-care workers, cosmetic procedures (such as tattooing and body piercing) and scarification (35; 36). Hepatitis $\mathrm{C}$ virus causes both acute and chronic infection. Acute HCV infection is occurred when presence of $\mathrm{HCV}$ within six months of exposure to HCV infection. The infection with $\mathrm{HCV}$ is usually clinically silent and is only very rarely associated with life-threatening disease. The acute $\mathrm{HCV}$ infection occurs within six months of infection in 15-45\% of infected pepeol in the absence of treatment, almost all the remaining $55-85 \%$ of persons will harbour $\mathrm{HCV}$ for the rest of their lives (if not treated) and are considered to have chronic HCV infection (37). Treatment is successful in the majority of persons treated, and treatment has high success rates among individuals treated in low- and middle-income countries are similar to those in high-income countries (38). Pepeol who become chronically infected are predicted to develop liver cirrhosis or hepatocellular carcinoma (39).

\section{Types of HCV Viral Load Tests: \\ There are two categories of $\mathrm{HCV}$ viral load tests:}

\section{Qualitative viral load tests:}

These tests determine the presence of $\mathrm{HCV}$ RNA in the blood. If viral RNA is detected, a positive result is reported; if viral RNA is not detected, the test result is negative.

\section{Quantitative viral load tests:}

These tests measure the amount of virus in one milliliter of blood. In the past the level of the viral load was correlated with the chances of responding to HCV treatment, but it is less important with the HCV directacting antiviral medications. Real time quantitative PCR test is very sensitive and can measure as few as $5-10 \mathrm{IU} / \mathrm{mL}$ (28).

\section{Alcoholic liver disease (ALD):}

In western countries, Alcohol is one of the most frequent cause of liver disease. Mortality due to liver cirrhosis in western countries is in direct proportion to alcohol consumption in France and Spain (over 30 deaths per a population of 100000 per year), the death in the northern European countries is lowest than western countries (up to 5 deaths per 100) (40).

However, there has been limited research investment into ALD despite its bad effect on the health of Europeans. In recent years however, the mechanisms driving disease progression and the natural history of ALD have been better defined and novel targets for therapy have been identified. In addition, significant clinical research has produced a clear framework for the evaluation of new therapies in particular in patients with alcoholic steatohepatitis (ASH) (41). Alcoholic liver disease (ALD) includes a spectrum of injury, ranging from 
simple steatosis to frank cirrhosis. It may well represent the oldest form of liver injury known to humanity. Evidence suggests that alcoholic beverages existed at least as early as the Neolithic period. Alcohol remains one of the most cause of liver disease worldwide, it is common for patients with ALD to share the risk factors for simultaneous injury from other liver insults (e.g., co-existing non-alcoholic fatty liver disease, or chronic viral hepatitis) (42).

\section{Nonalcoholic fatty liver disease:}

Non-alcoholic fatty liver disease (NAFLD) ranges from simple steatosis to nonalcoholic steatohepatitis (NASH), leading to fibrosis and may be cirrhosis (43).

Non-alcoholic fatty liver disease (NAFLD) is the most common cause of liver disease worldwide, with a spread of $15 \%-30 \%$ in Western populations (44). The prevalence of (NAFLD) increases to $58 \%$ in overweight people and increase to $98 \%$ in non-diabetic obese individuals (45).

NAFLD ranges from simple steatosis to nonalcoholic steatohepatitis (NASH) and potentially cirrhosis (46). It leads to hepatocellular injury and liver inflammation (47).

Cirrhosis, that occurs in $25 \%$ of patients with NASH, leads to liver failure, portal hypertension, and hepatocellular carcinoma and the patients with cirrhosis are at a high risk for developing cardiovascular disease (48). There are no clear data in terms of the incidence of NAFLD and NASH. As the incidence of all of the present factors is steadily increasing, the incidence of NAFLD and NASH is expected to increase rapidly worldwide. In the United States, for example, NASH is now believed as the most common cause of cirrhosis. Similar trends are reported for the rest of the Western World and even for developing countries (49).
Diet is an important reason to NAFLD, mainly because excessive calories intake leads to obesity, which increases the risk for NAFLD. However, not only the amount of energy but also the quality of the diet could play an important role for the development and progression of NAFLD. Diets rich in saturated fat, cholesterol, and low in polyunsaturated fat, fiber and antioxidant vitamins $\mathrm{C}$ and $\mathrm{E}$ have been associated with NASH. (50)

Other research has also shown a relationship between increased dietary fat intake and NAFLD (51). On the contrary, a study examines pre-surgical bariatric patients in the United States reported that increased carbohydrate intake was associated with hepatic inflammation (52). Especially fructose among carbohydrates might participate into NAFLD progression. Fructose intake leads to increasing hepatic fat, inflammation and may be fibrosis (53).

\section{Nonalcoholic fatty liver disease diagnosis:}

Most of the studies in the general population are based on liver ultrasound or liver chemistries, with liver biopsy mostly restricted to subjects at high risk for more aggressive liver disease. At least one third of the U.S. population is believed to have NAFLD (54; 55). A most recent group studies using ultrasound and liver biopsy fixed the propagation of NAFLD in asymptomatic middle-aged patients to be $46.0 \%$ and the dispersal of NASH to be $12.2 \%$ (56). The diagnosis of NAFLD requires confirmation of hepatic steatosis by imaging or liver biopsy (57). Ultrasound, computed tomography, or magnetic resonance studies can confirm the presence of hepatic steatosis with a relatively high degree of accuracy (58). 


\section{Cholinesterase enzyme:}

Animal cholinesterase are widespread enzymes present in cholinergic and noncholinergic tissues as well as in their plasma and other body fluids (59). Cholinesterase enzyme is present in mammals, belongs to hydrolases enzymes that catalyze hydrolysis and splitting of substrates by water. The reactions include also the cleavage of ester bonds in lipids. In general, larger molecules are broken down to smaller fragments by hydrolases as cholinesterase enzyme $(60 ; 61)$ and includes two classes: acetylcholinesterase (AChE) and butyrylcholinesterase (BuChE). AChE (true cholinesterase) is presented in the central nervous system, platelet and erythrocyte membranes while BuChE (pseudocholinesterase) is more abundant in the serum that is synthesized by the liver (62). AChE presents in brain, muscle and erythrocyte membrane, while $\mathrm{BChE}$ has higher activity in liver, intestine, heart, kidney and lung. Serum BChE is synthesized in liver and leave liver to plasma (63). The insecurity of acetylcholine (ACh) that presents in blood and tissues was appreciated even before it was positively identified as a transmitter By the early thirties of last century, this insecurity was recognized as being due to the presence of an enzyme which hydrolyzed $\mathrm{ACh}$ to the physiologically inactive choline and acetate, the enzyme had been given a name, choline-esterase, later shortened to cholinesterase (ChE), and it had been partially purified from serum (64). AChE hydrolyzes acetylcholine faster than other cholinesterase and is much less active on butyrylcholine. On the contrary, $\mathrm{BChE}$ preferentially acts on butyrylcholine, but also hydrolyzes acetylcholine (65). Pseudocholinesterase (BChE or BuChE), also known as plasma cholinesterase, butyrylcholinesterase, or (most formally) acyl choline acyl hydrolase is found primarily in the liver. The half-life of pseudocholinesterase is approximately816hours. Pseudocholinesterase levels may be decreased in patients with advanced liver disease. The reduction of BuChE must be greater than $75 \%$ before significant extention of neuromuscular blockade occurs with succinylcholine (66; 67). Clinically useful quantities of butyrylcholinesterase were synthesized in 2007 by Pharm Athene, through the use of genetically modified goats (68). The existence of the cholinesterase was predicted in 1914 by Dale, some 15 years before acetylcholine was shown to be a natural constituent of animal tissues. The term, 'cholinesterase' was first used in 1932 to depict an enzyme from horse serum which hydrolyzed acetylcholine, the substance liberated at the motor end plate during a nerve impulse. Hydrolysis then permitted the nerve fibre to accommodate the next impulse, in 1940 was appreciated that there are two enzymes which hydrolyze acetylcholine in human tissues, cholinesterase are among the fastest enzymes known with the hydrolysis of acetylcholine by acetylcholinesterase (69). Cholinesterase enzyme was removed at first time from electric tissue (70). Cholinesterase is an enzyme secreted by hepatocytes then leave into blood. Cholinesterase secretion is decreased in hepatocyte dysfunction in contrast to the increased release of other liver enzymes into the circulation indicating liver injury (71).

\section{Types of cholinesterase enzyme:}

Mammalian ChEs represent a family of enzymes that hydrolyse acetylcholine, and this enzyme are typically divided in two major classes: Acetylcholinesterase and butyrylcholinesterase, according to substrate preference and sensitivity to specific inhibitors AChE from mammalian 
tissues is characterized by the preference for acetylcholine (ASCh) hydrolysis, The hydrolysis of neurotransmitter acetylcholine at cholinergic synapses of central and peripheral nervous tissue is the main physiological role of AChE. BChE preferentially hydrolyze butyrylcholine although it can hydrolyze other choline esters as well (72). The role of BChE in the organism is still not ascertained, although it seems that it actually protects AChE by metabolisation of anti-cholinesterase compounds (73).

\section{Genetic structure of Cholinesterase:}

Vertebrates have two cholinesterase genes, encoding $\mathrm{AChE}$ and $\mathrm{BChE}$. In mammals, $\mathrm{AChE}$ is encoded by a single gene, that has to possess several transcriptions start sites (74). AChE and BChE share $65 \%$ amino acid sequence homology and have similar molecular forms and active center structure despite being products of different genes on human chromosomes 7 (specifically $7 \mathrm{q} 22$ ) and 3(specifically 3q26), respectively. The $\mathrm{AChE}$ main function is rapid hydrolysis of the neurotransmitter acetylcholine at cholinergic synapses, and it is one of the fastest enzyme known, when BChE is absent in some individuals does not correlate with any physiological abnormality, its importance as a detoxification enzyme is growing interest in recent years. BChE has pharmacological and toxicological importance, because it hydrolyzes ester-containing drugs and scavenges cholinesterase inhibitors including potent organophosphorus nerve agents before they reach their synaptic targets (63).

\section{Importance of Cholinesterase enzyme:}

Cholinesterase (ChE) is a family of enzymes that catalyze the hydrolysis of the neurotransmitter acetylcholine into choline and acetic acid, a reaction necessary to allow a cholinergic neuron to return to its resting state after activation (75). The neuromuscular junction connects the nervous system to the muscular system via synapses between efferent nerve fibers and muscle fibers, also known as muscle cells. As an action potential reaches the end of a motor neuron, voltage-dependent calcium channels open allowing calcium to enter the neuron. Calcium facilitates vesicle binding and subsequent neurotransmitter release from the motor neuron into the synaptic cleft. In vertebrates, motor neurons release acetylcholine (ACh), a small molecule neurotransmitter, which diffuses through the synapse and binds nicotinic acetylcholine receptors (nAChRs) on the plasma membrane of the muscle fiber, also known as the sarcolemma. During neurotransmission, $\mathrm{ACh}$ is released from the nerve into the synaptic cleft and binds to ACh receptors on the post-synaptic membrane, relaying the signal from the nerve. AChE, also located on the postsynaptic membrane, terminates the signal transmission by hydrolyzing ACh (Figure 1). The liberated choline is taken up again by the pre-synaptic nerve and $\mathrm{ACh}$ is synthetized by combining with acetyl-CoA through the action of choline acetyl transferase (76). 


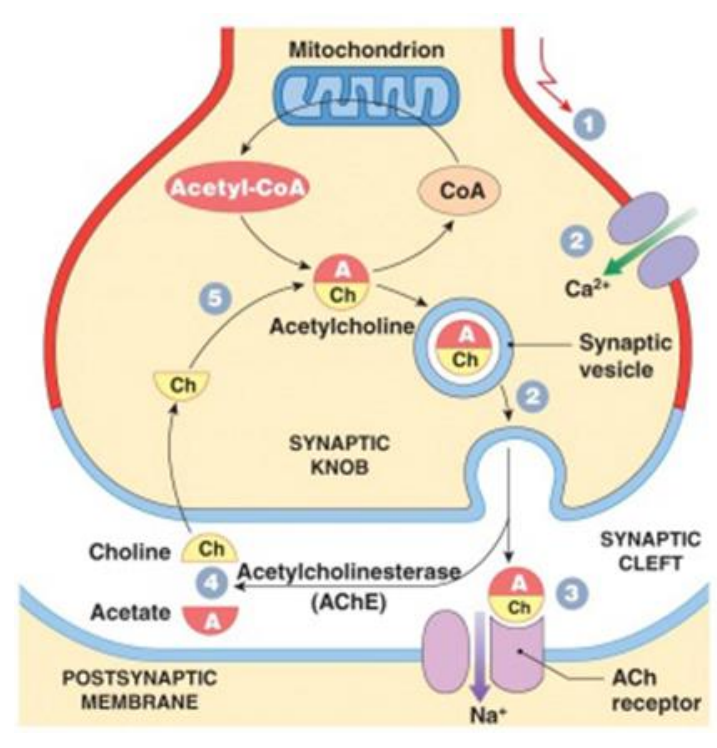

Figure 1. Role of acetylcholinesterase enzyme during neurotransmission.

\section{Acetylcholine synthesis:}

$\mathrm{ACh}$ is synthesized by choline acetyltransferase (ChAT) and is loaded into synaptic vesicles by the vesicular acetylcholine transporter (VAChT). The synaptic vesicle lumen is acidified by the action of an ATP-dependent proton pump located in the synaptic vesicle membrane. The action of the transmitter is terminated by transportermediated removal of the transmitter from the synaptic cleft. The action of acetylcholine, however, is terminated by direct enzymatic hydrolysis of the neurotransmitter in the synaptic cleft by acetylcholinesterase (AChE) (Figure 2). The resulting choline is then transported back into the presynaptic neuron by a high affinity choline transporter (HAChT, or ChT); this choline is then available for the synthesis of additional ACh (77). butyrylcholinesterase rapidly sequesters organophosphorus (OP) compounds by stoichiometric binding and hydrolyzes cocaine and the muscle relaxants succinylcholine and mivacurium, which make it suitable for many prophylactic and therapeutic applications. Exogenously administered purified $\mathrm{Hu}$ BChE was successfully shown to protect animals from toxicity of OP pesticides and nerve agents and for treating the toxic effects of cocaine (78).

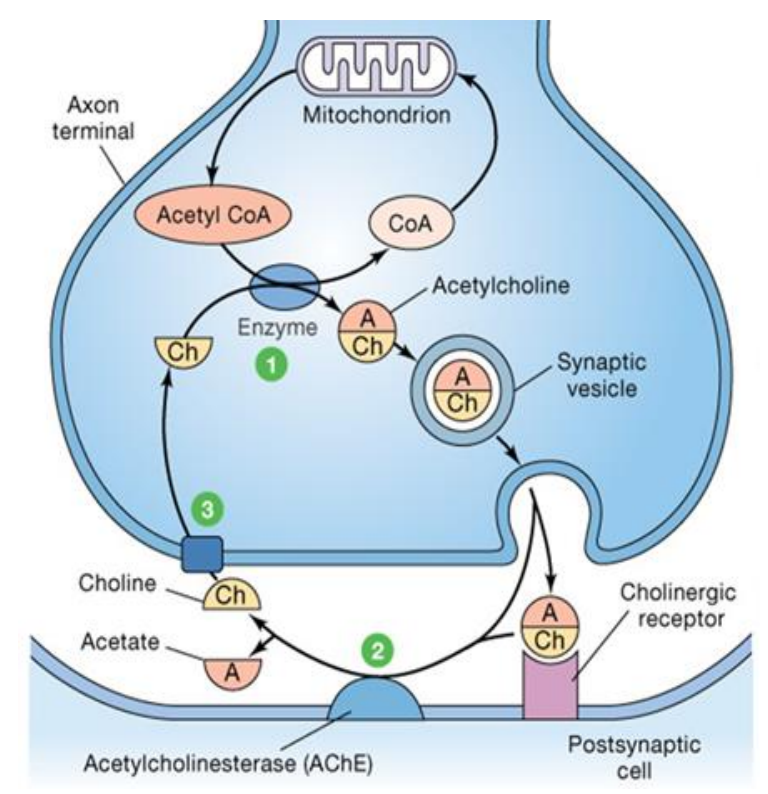

Figure 2. Acetylcholine synthesis.

\section{Micro RNA:}

MicroRNAs are important regulators of gene expression that control all physiological and pathological processes like development and cancer. Although their mode of action has attracted great attention, the principles governing their expression and activity are only beginning to emerge. Studies at last years have introduced a model shift in our understanding of the microRNA biogenesis pathway, that was once upon time believed to be universal to all micro RNAs. Maturation steps specific to individual microRNAs have been uncovered, and these offer a plethora of regulatory options after transcription with multiple proteins affecting microRNA processing efficiency. Here we review the recent advances in knowledge of the microRNA biosynthesis pathways and discuss their impact on post- 
transcriptional microRNA regulation during tumor development (79).

\section{Small RNA:}

To prevent equivocation, a distinction must be present between microRNA and siRNAs (Small interfering RNA, sometimes known as short interfering RNA or silencing RNA). Micro RNA are short, endogenous RNAs derived from single-stranded precursor RNAs fashioned with an imperfectly base paired hairpin segment. siRNAs, on the other hand, are similar in length but are derived from longer, perfectly complementary double-stranded RNA precursors of mainly exogenous origin $(80 ; 81)$. Both mature through a duplex intermediate, with unpaired 3_ extensions and a 5_ phosphate. Functionally, microRNA tend not to be exactly complementary with their targets, because of the presence of mismatches and bulges, whereas siRNAs are usually exactly complementary. This seems to be important for their mechanism of action, as is discussed below. However, if a microRNA is perfectly matched with its target, it can act as an siRNA and, similarly, an imperfectly matched siRNA can act as if it were a microRNA. Thus, MicroRNA and siRNA cannot be distinguished by mechanism but only through their origins and biogenesis $(82 ; 83)$. Bioinformatics approaches have been developed to predict putative miRs present in the genome of different organisms, based on the fact that they are usually highly conserved between related species and produced from precursor transcripts of similar size and structure. The first microRNA search algorithm to be developed was microRNA scan (84), whereas other algorithms such as PromiR II 34 and PalGrade35 were specifically designed to be used for humans $(85 ; 86)$. Newly identified microRNA are denoted with sequential numbers unless they are orthologs (homologous microRNA in different organisms), in which case the same number is used. Now, predicted 1000 microRNA may exist in the human genome (87). Currently, around $796 \mathrm{miR}$ human sequences have been identified and have been catalogued in a searchable Web based data registry (88).

\section{Structure and function of microRNA:}

MicroRNA (miRNA) (MiRs) is short ( 22 nucleotides), non-coding RNAs, (Figure 3) that regulate various molecular pathways $(89 ; 90)$. MicroRNA was first discovered in 1993, and was characterized as a small, non-coding RNA molecule, lin-4 (6). About a few years later the second miRNA, let 7 was discovered (91). These two discoveries of miRNA support further studies on discovery of new miRNAs, and so a large class of small non-coding RNAs emerged with a diverse range of biological functions, such as temporal regulation of development, cell death and proliferation, hematopoiesis and tumorigenesis $(82 ; 92)$.

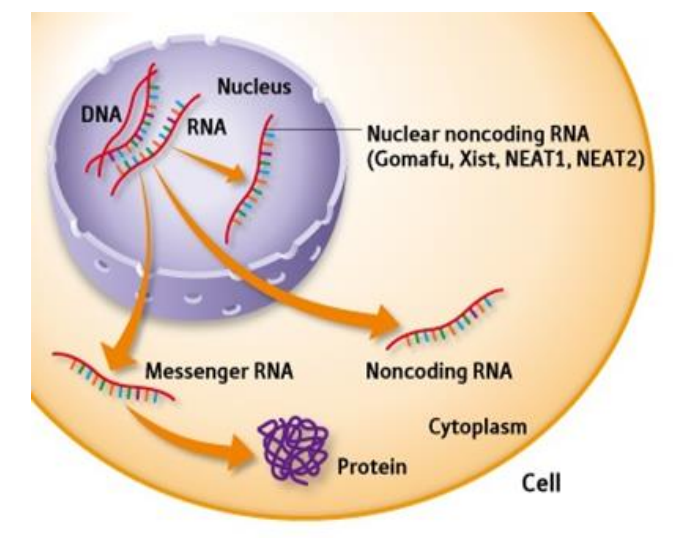

Figure 3. Noncoding Micro RNA.

Thousands of miRNAs have been found in animals, plants, viruses and an individual miRNA can post-transcriptionally target hundreds of mRNAs $(93 ; 94)$. Untill this time more than 700 human miRNAs have 
been cloned, also it has been estimated that more than 45,000 miRNA target sites are present within human, and that more than 60 percent of human protein-coding genes are possibly regulated by miRNAs, in the animal kingdom, about 30 percent of all genes are targeted by miRNA (95). MiRNAs detection led to a worldwide research effort to clear their roles in cancer. miRNAs regulate molecular pathways in cancer by targeting various oncogenes and tumour suppressors and have a role in cancer and stem cell biology, angiogenesis, the epithelial-mesenchymal transition, metastasis, and drug resistance (96). The significance of miRNAs and the small RNA (sRNAs) has been found across the living kingdom, including their roles in regulating immune response and host-pathogen interactions during bacterial, fungal and viral infections, and response to environmental stress have been reviewed elsewhere (97).

\section{Mechanisms of Function of microRNAs:}

miRNAs are mainly negative regulators of mRNA translation and exert their effects through various mechanisms as a first step, the pre-miRNAs are assembled in large protein complexes known as miRNA RNAinducing silencing complex (RISC or miRISC) (98). An exposition of the steps and molecules involved in the processing and function of miRNAs. The first step does in the nucleus, where pre-miRNA is cleaved via the enzymes Drosha and Pasha. The pre-miRNA hairpin structure is then exported to the cytoplasm. In the cytoplasm it assembles in RISC which includes Dicer(that is a human enzyme cleaves double-stranded RNA (dsRNA) and premicroRNA into short double-stranded RNA fragments called small interfering RNA and microRNA respectively), argonaute (Ago) protein (proteins that are the catalytic components of RNA-induced silecing complex (RISC)), PW182 a P-body protein(it is the proteins necessary for miRNA gene silencing are localized to Pbodies), the human immunodeficiency virus transactivating response RNAbinding protein (TRBP), and fragile $\mathrm{X}$ mental retardation protein (FMRP1) (this protein, most commnly found in the brain, is essential for normal cognitive development and reproductive function. Mutation of this gene can lead to fragile X syndrome, mental retardation, premature ovarien failure). In RISC, the loop of the pre-miRNAs is cleaved, and the mature strand is used to direct the complex to its target site in of an mRNA. RISC is involved in 3 different functions: inhibition of translation initiation, inhibition of translation elongation, or mRNA deadenylation, which would result in mRNA degradation. These functions require additional recruitment of eukaryotic initiation factor 6 (eIF6). Inhibition of translation initiation is a result of disengagement of the $80 \mathrm{~S}$ ribosomal complex in a cap (m7GpppG)-dependent manner that is partly attributed to the recruitment of the anti-association factor eIF6 by the RISC complex, or competition of Ago2 with eukaryotic initiation factor $4 \mathrm{E}$ (eIF4E) or eIF4G. Deadenylation occurs via interaction of Ago2 with chromatin assembly factor 1 (CAF1) and, thus, recruitment of the CCR4-NOT protein complex that harbors both chemokine (C-C motif) receptor (CCR4) and CAF1 deadenylase enzymes, in addition to a second deadenylation step involving the PABP-dependent poly(A) nuclease 2 (Pan2)-Pan3 deadenylase complex (99). 


\section{References}

1. Lauer GM, Walker BD. Hepatitis C virus infection. N Engl J Med, 345: 41$52,2001$.

2. Tauber SC, Nau R. Immunomodulatory properties of antibiotics. Current Molecular Pharmacology. 2008;1(1): $86-79$.

3. Lai AY, Todd KG. Hypoxia-activated microglial mediators of neuronal survival are differentially regulated by tetracyclines. GLIA. 2006;53(8):809816.

4. X. Castro, J. E. Z., Vado-Solis, I., Perez-Osorio, C., \& Fredeking, T. M. (2011). Modulation of Cytokine and Cytokine Receptor/Antagonist by Treatment with Doxycycline and Tetracycline in Patients with Dengue Fever. Clinical and Developmental Immunology, 2011, 1-5. Science China Life Sciences., 63 (3) (2020), pp. 457 460.

5. Lemaitre M, Guetard D, Henin $Y$, Montagnier L, Zerial A. Protective activity of tetracycline analogs against the cytopathic effect of the human immunodeficiency viruses in CEM cells. Res Virol 1990; 141:5-16.

6. Szeto GL, Brice AK, Yang HC, Barber SA, Siliciano RF, Clements JE. Minocycline attenuates HIV infection and reactivation by suppressing cellular activation in human CD4+ T cells. $\mathrm{J}$ Infect Dis 2010; 201:1132-40.

7. Atrasheuskaya AV, Fredeking TM, Ignatyev GM. Changes in immune parameters and their correction in human cases of tick-borne encephalitis.
Clinical and Experimental Immunology 2003;131(1):148-154.

8. Bethell DB, Flobbe K, Phuong CXT, et al. Pathophysiologic and prognostic role of cytokines in dengue hemorrhagic fever. Journal of Infectious Diseases. 1998;177(3):778782.

9. Green S, Vaughn DW, Kalayanarooj S, et al. Early immune activation in acute dengue illness is related to development of plasma leakage and disease severity. Journal of Infectious Diseases. 1999;179(4):755-762.

10. Girardin E, Roux-Lombard P, Grau GE, Suter P, Gallati H, Dayer J-M. Imbalance between tumour necrosis factor-alpha and soluble TNF receptor concentrations in severe meningococcaemia Immunology. 1992;76(1):20-23.

11. Van Dissel JT, van Langevelde $P$, Westendorp RGJ, Kwappenberg K, Frölich M. Anti-inflammatory cytokine profile and mortality in febrile patients. Lancet. 1998;351(9107):950953.

12. Dinarello CA. Anti-cytokine therapeutics and infections. Vaccine. 2003;21(2): S24-S34.

13. Clark IA, Budd AC, Alleva LM, Cowden WB. Human malarial disease: a consequence of inflammatory cytokine release. Malaria Journal. 2006; 5:85-117.

14. Bozza FA, Cruz OG, Zagne SMO, et al. Multiplex cytokine profile from dengue patients: MIP-1beta and IFN-gamma as predictive factors for severity. BMC Infectious Diseases. 2008; 8:86-93. 
15. Green S, Vaughn DW, Kalayanarooj S, et al. Early immune activation in acute dengue illness is related to development of plasma leakage and disease severity. Journal of Infectious Diseases. 1999;179(4):755-762.

16. Castro, J. E. Z., Vado-Solis, I., PerezOsorio, C., \& Fredeking, T. M. (2011). Modulation of Cytokine and Cytokine Receptor/Antagonist by Treatment with Doxycycline and Tetracycline in Patients with Dengue Fever. Clinical and Developmental Immunology, 2011, $1-5$.

17. Yang JM, Chen YF, Tu YY, Yen KR, Yang YL. Combinatorial computational approaches to identify tetracycline derivatives as flavivirus inhibitors. PLoS ONE. 2007;2(5): e428-e441.

18. X.N. Tang, Q. Wang, M.A. Koike, et al., Monitoring the protective effects of minocycline treatment with radiolabeled annexin $\mathrm{V}$ in an experimental model of focal cerebral ischemia, J. Nucl. Med. 48 (11) (2007) 1822-1828.

19. Giuliani F, Hader W, Yong VW. Minocycline attenuates $\mathrm{T}$ cell and microglia activity to impair cytokine production in $\mathrm{T}$ cell-microglia interaction. J Leukoc Biol 2005; 78:135-43.

20. Popovic N, Schubart A, Goetz BD, Zhang SC, Linington C, Duncan ID. "Inhibition of autoimmune encephalomyelitis by a tetracycline ". Ann Neurol 2002; 51:215-23.

21. Song Y, Wei EQ, Zhang WP, Zhang L, Liu JR, Chen Z. Minocycline protects PC12 cells from ischemic-like injury and inhibits 5- lipoxygenase activation. NeuroReport 2004; 15:2181-4.

22. Chen M, Ona VO, Li M, Ferrano RJ, Fink KB, Zhu S. minocycline inhibits caspase - and caspase -3 expression and delays mortality in a transgenic mouse model of Huntington disease. Nat Med 2000; 6:797-801.

23. Domerq M, Matute C. Neuroprotection of by tetracyclines. Trends Pharmacol Sci 2004; 25:609-12.

24. Szeto GL, Brice AK, Yang HC, Barber SA, Siliciano RF, Clements JE. Minocycline attenuates HIV infection and reactivation by suppressing cellular activation in human CD4+ $\mathrm{T}$ cells. $\mathrm{J}$ Infect Dis 2010; 201:1132-40.

25. J. Yrjänheikki, T. Tikka, R. Keinänen, G. Goldsteins, P.H. Chan, J. Koistinaho, Atetracycline derivative, minocycline, reduces inflammation and protects againstfocal cerebral ischemia with a wide therapeutic window, Proc. Natl. Acad. Sci. U. S.A. 96 (23) (1999) 13496-13500.

26. K. Hayakawa, K. Mishima, M. Nozako, et al., Delayed treatment with minocyclineameliorates neurologic impairment through activated microglia expressing a high-mobility group box 1inhibiting mechanism, Stroke 39 (3) (2008) 951-958.

27. T. Tikka, B.L. Fiebich, G. Goldsteins, R. Keinanen, J. Koistinaho, Minocycline, atetracycline derivative, is neuroprotective against excitotoxicity by inhibiting ac-tivation and proliferation of microglia, $\mathrm{J}$. Neurosci. 21 (8) (2001) 2580-2588. 
28. X.N. Tang, Q. Wang, M.A. Koike, et al., Monitoring the protective effects of mino-cycline treatment with radiolabeled annexin $\mathrm{V}$ in an experimental model of focalcerebral ischemia, J. Nucl. Med. 48 (11) (2007) 1822-1828.

29. R.O. Sanchez Mejia, V.O. Ona, M. Li, R.M. Friedlander, Minocycline reduces trau-matic brain injury-mediated caspase- 1 activation, tissue damage, and neurologicaldysfunction, Neurosurgery 48 (6) (2001) 13931399.

30. Mohit Sodhi and Mahyar Etminan, Letter to the Editor, Therapeutic Potential for Tetracyclines in the Treatment of COVID-19.

31. Zakeri B, Wright GD. Chemical biology of tetracycline antibiotics. Biochem Cell Biol. 2008;86(2):124136.

32. Humar, A., McGilvray, I., Phillips, M.J. and Levy, G.A. (2004), Severe acute respiratory syndrome and the liver. Hepatology, 39: 291-294.

33. Phillips JM, Gallagher T, Weiss SR. Neurovirulent Murine Coronavirus JHM.SD Uses Cellular Zinc Metalloproteases for Virus Entry and Cell-Cell Fusion. J Virol. 2017;91(8): e01564-16

34. Rothan HA, Mohamed Z, Paydar M, Rahman NA, Yusof R. Inhibitory effect of doxycycline against dengue virus replication in vitro. Arch Virol. 2014; 159(4):711-718.

35. Sturtz FG. Antimurine retroviral effect of doxycycline. Methods Find Exp Clin Pharmacol. 1998; 20(8):643-647.
36. Henehan M, Montuno M, De Benedetto A. Doxycycline as an antiinflammatory agent: updates in dermatology. J Eur Acad Dermatol Venereol. 2017; 31(11):1800-1808.

37. Yoshikawa T, Hill T, Li K, Peters CJ, Tseng CT. Severe acute respiratory syndrome (SARS) coronavirus-induced lung epithelial cytokines exacerbate SARS pathogenesis by modulating intrinsic functions of monocyte-derived macrophages and dendritic cells. J Virol. 2009; 83(7):3039-3048.

38. Kritas SK, Ronconi G, Caraffa A, Gallenga CE, Ross R, Conti P. Mast cells contribute to coronavirus-induced inflammation: new anti-inflammatory strategy [published online ahead of print, 2020 Feb 4]. J Biol Regul Homeost Agents. 2020; 34(1):10.23812/20- Editorial-Kritas.

39. Sandler C, Nurmi K, Lindstedt KA, et al. Chemically modified tetracyclines induce apoptosis in cultured mast cells. Int Immunopharmacol. 2005;5(11):1611-1621.

40. Sandler C, Ekokoski E, Lindstedt KA, et al. Chemically modified tetracycline (CMT)-3 inhibits histamine release and cytokine production in mast cells: possible involvement of protein kinase C. Inflamm Res. 2005;54(7):304-312

41. Griffin MO, Fricovsky E, Ceballos G, Villarreal F. Tetracyclines: a pleitropic family of compounds with promising therapeutic properties. Review of the literature. Am J Physiol Cell Physiol. 2010;299(3):C539-C548 
